\title{
A Multicenter Study on Expressions of Vascular Endothelial Growth Factor, Matrix Metallopeptidase-9 and Tissue Inhibitor of Metalloproteinase-2 in Oral and Maxillofacial Squamous Cell Carcinoma
}

\author{
Min Li ${ }^{1}$; Zhiying Wang ${ }^{1, *}$; Yang Xing ${ }^{2}$; jin Yu ${ }^{3}$; Luming Tian ${ }^{4}$; Dianming Zhang ${ }^{5}$; Zengxi Xin ${ }^{3}$ \\ ${ }^{1}$ Department of Oral Implantology, Second Affiliated Hospital of Liaoning Medical University, Jinzhou, China \\ 2 Jinzhou City Oral Cavity Hospital, Jinzhou, China \\ 3 Department of Prosthodontics, Second Affiliated Hospital of Liaoning Medical University, Jinzhou, China \\ ${ }_{5}^{4}$ Department of Oral and Maxillofacial Surgery, Second Affiliated Hospital of Liaoning Medical University, Jinzhou, China \\ 5 Department of Oral and Maxillofacial Surgery, Second Affiliated Hospital of Liaoning Medical \\ *Corresponding Author: Zhiying Wang, Department of Oral Implantology, Second Affiliated Hospital of Liaoning Medical University, Jinzhou 121001, P. R. China. Tel: +86-4162332215, \\ Fax:+86-4162332215, E-mail: wangzhiyingdoi@163.com
}

Received: June 25, 2013; Revised: December 11, 2013; Accepted: January 29, 2014

\begin{abstract}
Background: Vascular endothelial growth factor (VEGF), matrix metallopeptidase-9 (MMP-9) and tissue inhibitor of metalloproteinase-2 (TIMP-2) are potential markers of oral and maxillofacial squamous cell carcinoma (SCC).

Objectives:To explore the association between expression of VEGF, MMP-9 and TIMP-2 in oral and maxillofacial SCCand clinicopathological factors.

Patients and Methods: Immunohistochemical Envision method was used to analyze the expression of VEGF, MMP-9 and TIMP-2 in 54 cases of oral and maxillofacial SCC and the association with clinicopathological factors such as clinical staging and lymphatic metastasis. Results: Brownish-yellow staining is correlated with positive expression of VEGF, MMP-9 and TIMP-2. Positive expression of VEGF and MMP-9 was correlated with lymphatic metastasis, and their positive expression rates were significantly higher in patients with lymphatic metastasis than those without it $\left(\mathrm{VEGF}: \chi^{2}=30.00 ; \mathrm{P}=0.001\right.$;MM-9: $\left.\chi^{2}=18.27, \mathrm{P}=0.001\right)$. The positive expression rate of MMP-9 decreased at earlier clinical stages $(\mathrm{P}<0.05)$. Positive expression of TIMP-2 was correlated with lymphatic metastasis, clinical staging and Tclassification. The positive rate of TIMP-2 expression in patients with lymphatic metastasis was significantly lower than those without it $\left(\chi^{2}=26.74, \mathrm{P}=\right.$ $0.002)$, which significantly reduced with increasing clinical stage and T classification $(\mathrm{P}<0.05)$.

Conclusions: Lymphatic metastasis in patients with oral and maxillofacial SCC is closely related to the positive expression of VEGF, MMP-9 and TIMP-2. MMP-9 and TIMP-2 can affect the progression of cancer, which is valuable for studies on oral and maxillofacial SCC genes.
\end{abstract}

Keywords:Oral Surgical Procedures; Carcinoma, Squamous Cell; Vascular Endothelial Growth Factor A; Matrix Metalloproteinase

9; Tissue Inhibitor of Metalloproteinase-2

\section{Background}

Maxillofacial malignant tumors are mainly carcinomas instead of sarcomas in China. Among carcinomas, the incidence rate of squamous cell carcinoma (SCC) ranks first at about $80 \%(1,2)$. Currently, oral and maxillofacial SCC is mainly treated by surgery and radiotherapy, but the effect is unsatisfactory, thus demanding targeted therapy. Tumor stem cells in oral and maxillofacial SCC, such as vascular endothelial growth factor (VEGF), phosphatase and tensin homolog (PTEN), matrix metallopeptidase-9 (MMP-9), tissue inhibitor of metalloproteinase-2 (TIMP2), CD44 and CD133, have been potential markers (3) by exerting corresponding effects on the occurrence and suppression of tumors or lymphatic metastasis. Tumor markers (4), produced by tumors themselves, include embryonic antigen, carbohydrate antigen, related enzymes, hormones and gene, and some others. The content changes of the markers indicate the properties of tumors, providing references for further treatment by preliminarily suggesting the changes of tumor tissues as well as the differentiation and function of cells. For instance, the markers for lung cancer are CEA, CYFRA21-1 and NSE, and those for prostate cancer are f-PSA and t-PSA. However, the markers often function through the synergetic effects of molecules such as CD44 and CD133, PTEN

Implication for health policy/practice/research/medical education:

Lymphatic metastasis in patients with oral and maxillofacial squamous cell carcinoma (SCC) is closely related to positive expressions of VEGF, MMP-9 and TIMP-2. Meanwhile, MMP-9 and TIMP-2 can also affect the progression of cancer, which is of great significance to studies on oral and maxillofacial SCC genes.

Copyright (C) 2014, Iranian Red Crescent Medical Journal; Published by Kowsar Corp. This is an open-access article distributed under the terms of the Creative Commons Attribution License, which permits unrestricted use, distribution, and reproduction in any medium, provided the original work is properly cited. 
and MMP-9, and some others, contributing to the advent of "gene synergistic hypothesis".

\section{Objectives}

This study aimed to investigate the expression levels of VEGF, MMP-9 and TIMP-2 in 54 patients with oral and maxillofacial SCC in our medical institutions based on gene synergistic hypothesis to explore its association with clinicopathological factors.

\section{Patients and Methods}

\subsection{Clinical Information}

The human research ethics committees of our institutions approved the study, and an informed consent was obtained from all participants. Sixty patients with maxillofacial SCC were treated from April 2011 to April 2013, of which 54 cases with primary foci and without metastases to other organs were included in this retrospective clinical study, consisting of 40 males and 14 females aged 26 - 65 years old with an average age of 50.3. All the included patients had complete records of clinical data. There were 6 cases of tongue cancer, 6 cases of floor-of-mouth cancer, 33 cases of cheek cancer and 10 cases of gum cancer. According to the TNM staging criteria for oral squamous cell carcinoma, there were 26 cases of T1-T2 stage and 28 cases of T3-T4 stage. According to the clinical staging criteria established by UICC, there were 18 cases of I-II stage and 36 cases of III-IV stage.

\subsection{Sampling Strategy}

\subsubsection{Sampling Center}

The observer of this study, Dr. Lili Wang, who is not one of the coauthors, is actually affiliated to Second Affiliated Hospital of Liaoning Medical University (Jinzhou, China) and PLA General Hospital (Beijing, China). Therefore, the clinical data in this study was collected from two locations (Jinzhou and Beijing) and the pathological departments of more than three medical institutions.

\subsubsection{Sampling Guideline}

Primary maxillofacial SCC; without metastases; complete clinical data; informed consent was obtained from all enrolled research participants

\subsubsection{Sampling Methods}

The mucosae of the 54 cases with lesions were sampled, fixed in $10 \%$ formaldehyde, embedded with paraffin, and prepared into sections.

\subsection{Immunohistochemical Envision Two-Step Staining}

\subsubsection{Main Materials}

Envision Flex (+) detection kit and ready-to-use (RTU) primary antibody reagent used in Envision method were purchased from Beijing XiYa JinQiao Biological Technology Co., Ltd.; horseradish catalase DAB chromogenic reagent kit was obtained from Tianjin Baihao Biological Technology Co., Ltd.; polylysine solution was obtained from Fuzhou Maixin Biological Technology Development Co., Ltd.

VEGF and MMP-9 mouse anti-human monoclonal antibodies were provided by Shanghai Huayi Biological Technology Co., Ltd. TIMP-2 rabbit anti-human polyclonal antibody was obtained from Beijing Zhongshan Golden Bridge Biotechnology Co., Ltd.

\subsubsection{Two-Step Staining Method}

Paraffin sections of cancer cells were placed in a $67^{\circ} \mathrm{C}$ incubator for deparaffinage, taken out after about $2 \mathrm{~h}$, and then washed with PBS ( $\mathrm{pH} 7.4$ ) three times (3 min/ time) (4-7). The sections were placed onto plastic shelves, placed into microwave-boiling citrate buffer ( $\mathrm{pH}$ 6.0), taken out after boiling by medium wave for about 10 min, cooled by flowing water, and washed with PBS twice (3 min/time). Each section was drop wise added $\mathrm{H}_{2} \mathrm{O}_{2}$, and washed with PBS three times at room temperature after $10 \mathrm{~min}$ (3 min/time). Then PBS was removed, and each section was added primary antibody and incubated at room temperature, and washed with PBS five times after $2 \mathrm{~h}$ (3 $\mathrm{min} /$ time). PBS was removed again, and each section was added avidin, incubated for $20 \mathrm{~min}$, and then washed with PBS. After PBS was removed, each section was added polymer enhancer and washed after $20 \mathrm{~min}$. After removal of PBS, each section was added appropriate anti-mouse/ rabbit polymer, incubated for 30 min and washed with PBS. After PBS was removed again, and each section was added DAB and observed by microscope. After hematoxylin counter staining, $\mathrm{HCl}$ differentiation, bluing after tap water washing, drying and clearing, sections were sealed with vegetable gum and observed after being dried in air.

\subsection{Observation Indices}

\subsubsection{Positive Expression}

VEGF, MMP-9 and TIMP-2 were regarded as positive expressions in oral and maxillofacial SCC when sections showed brownish yellow after staining. Yellow particulate matters appeared in the cytoplasm (8) could be ob- 
served by microscope. At the same time, primary antibody was replaced with PBS as the negative control. Five cell visual fields were selected, with about 200 cells in each field on average to calculate the percentage of positive tumor cells to total ones. The percentage of positive cells equal to or higher than $10 \%$ was considered positive $(+)$, and the opposite indicated negative (-) (9).

\subsubsection{Clinicopathological Factors}

Preoperative and postoperative cervical lymph node dissection samples were observed after HE staining (10), and sent to pathology laboratory for expert assistance to determine the occurrence of lymphatic metastasis. Clinical records of patients were reviewed to analyze the association between the positive expressions of VEGF, MMP-9 and TIMP-2 in oral and maxillofacial SCC and clinical staging and $\mathrm{T}$ classification.

\subsection{Statistical Analysis}

All data was analyzed by SPSS 17.0 and subjected to Chi Square test. $\mathrm{P}<0.05$ was considered statistically significant.

\section{Results}

\subsection{VEGF Expression}

Positive expression of VEGF was associated with lymphatic metastasis, and the positive rate of VEGF expression was significantly higher in patients with lymphatic metastasis than those without it $\left(\chi^{2}=30.00, \mathrm{P}=0.001\right)$. Positive VEGF expression had no association with gender, age, cancerous part, clinical staging and T classification $(\mathrm{P}>0.05)$ (Table 1, Figure 1)

\subsection{MMP-9 Expression}

Positive expression of MMP-9 was related with lymphatic metastasis, and the positive rate of MMP-9 expression was significantly higher in patients with lymphatic metastasis than those without it $\left(\chi^{2}=18.27, \mathrm{P}=0.001\right)$. Meanwhile, the positive expression rate of MMP-9 increased with increasing clinical staging $\left(\chi^{2}=7.96, \mathrm{P}=0.028\right)$. Its positive expression had no association with gender, age and other factors $(\mathrm{P}>0.05)$ (Table 2, Figure 2).

\begin{tabular}{|c|c|c|c|c|c|}
\hline Related factor & $\mathbf{n}$ & Positive (+) & Negative (-) & $\chi^{2}$ & P Value \\
\hline Gender & & & & & $0.832^{b}$ \\
\hline Male & 40 & $22(55.0)$ & $18(45.0)$ & 0.02 & \\
\hline Female & 14 & $8(57.1)$ & $6(42.9)$ & & \\
\hline Age, y & & & & & $0.376^{b}$ \\
\hline $26-50$ & 25 & $14(56.0)$ & $11(44.0)$ & 0.00 & \\
\hline $50-65$ & 29 & $16(55.2)$ & $13(44.8)$ & & \\
\hline Cancerous part & & & & & $0.921^{b}$ \\
\hline Tongue cancer & 6 & $2(33.3)$ & $4(66.7)$ & 0.01 & \\
\hline $\begin{array}{l}\text { Floor-of-mouth } \\
\text { cancer }\end{array}$ & 6 & $3(50.0)$ & $3(50.0)$ & & \\
\hline Cheek cancer & 33 & $19(57.6)$ & $14(42.4)$ & & \\
\hline Gum cancer & 10 & $6(60.0)$ & $4(40.0)$ & & \\
\hline Clinical stage & & & & & $>0.999^{b}$ \\
\hline I - II & 18 & $10(55.6)$ & $8(44.4)$ & 0.00 & \\
\hline III - IV & 36 & $20(55.6)$ & $16(44.4)$ & & \\
\hline T classification & & & & & $0.982^{b}$ \\
\hline $\mathrm{T} 1-\mathrm{T} 2$ & 26 & $15(57.7)$ & $11(42.3)$ & 0.09 & \\
\hline $\mathrm{T} 3-\mathrm{T} 4$ & 28 & $15(53.6)$ & $13(46.4)$ & & \\
\hline $\begin{array}{l}\text { Lymphatic metas- } \\
\text { tasis }\end{array}$ & & & & & $0.001^{c}$ \\
\hline Yes & 27 & $25(92.6)$ & $2(7.4)$ & 30.00 & \\
\hline No & 27 & $5(18.5)$ & $22(81.5)$ & & \\
\hline
\end{tabular}

a Data are presented as No.(\%).

b Without significant differences.

c With Significant Difference. 
Li M et al.
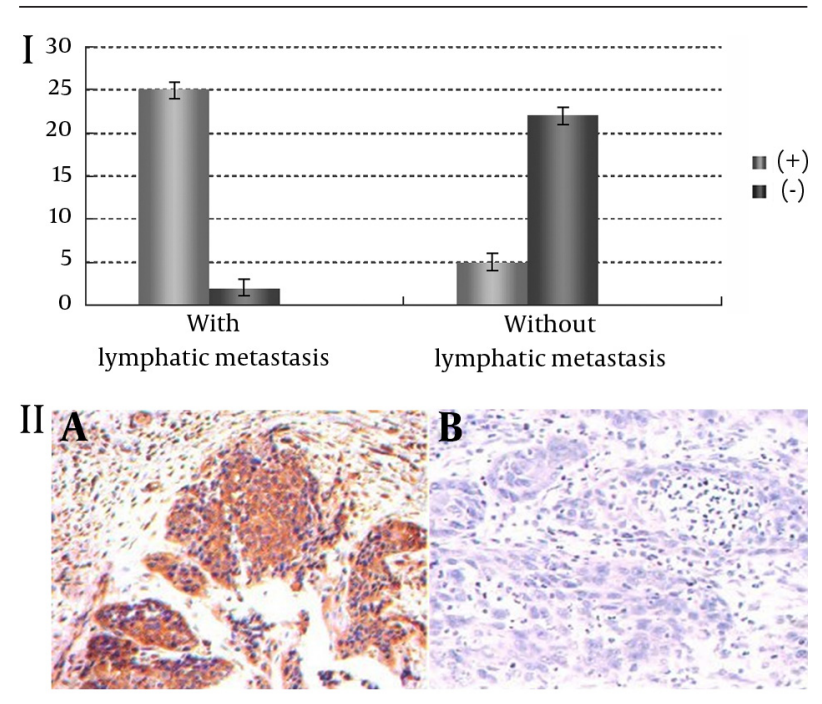

Figure 1. I: Association between VEGF positive expression and lymphatic metastasis; II: expression of VEGF in lymph nodes. A: With lymphatic metastasis, positive expression (+); B: Without lymphatic metastasis, negative expression (-).
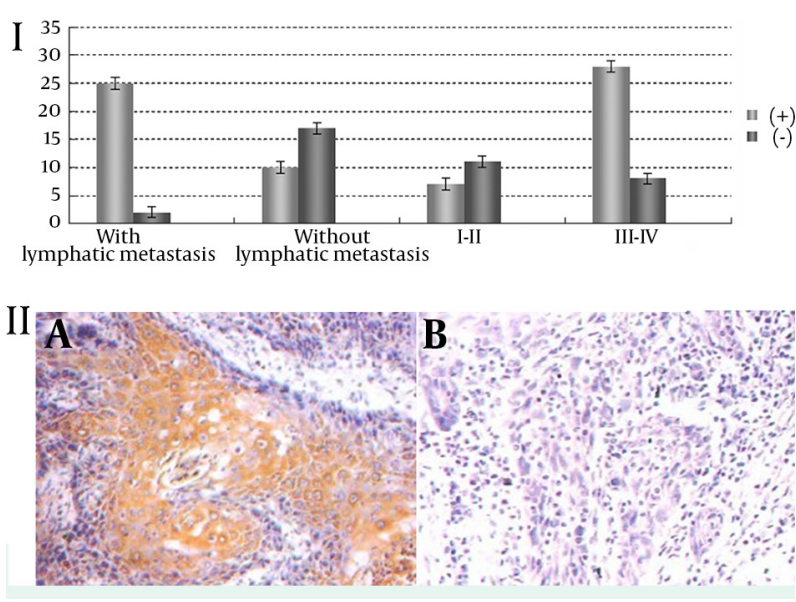

Figure 2. I: Association between MMP-9 positive expression and lymphatic metastasis and clinical stages; II: expression of MMP-9 in lymph nodes. A: With lymphatic metastasis, positive expression (+); B: Without lymphatic metastasis, negative expression (-).

\begin{tabular}{|c|c|c|c|c|c|}
\hline Related Factor & $\mathbf{n}$ & Positive (+) & Negative (-) & $\chi^{2}$ & P Value \\
\hline Gender & & & & & $0.737^{b}$ \\
\hline Male & 40 & $25(62.5)$ & $15(37.5)$ & 0.36 & \\
\hline Female & 14 & $10(71.4)$ & $4(28.6)$ & & \\
\hline Age & & & & & $0.821^{b}$ \\
\hline $26-50$ & 25 & $17(68.0)$ & $8(32.0)$ & 0.21 & \\
\hline $50-65$ & 29 & $18(62.1)$ & $11(37.9)$ & & \\
\hline Cancerous part & & & & & $0.691^{b}$ \\
\hline Tongue cancer & 6 & $5(83.3)$ & $1(16.7)$ & 0.26 & \\
\hline $\begin{array}{l}\text { Floor-of-mouth } \\
\text { cancer }\end{array}$ & 6 & $4(66.7)$ & $2(33.3)$ & & \\
\hline Cheek cancer & 33 & $20(60.6)$ & $13(39.4)$ & & \\
\hline Gum cancer & 10 & $6(60.0)$ & $4(40.0)$ & & \\
\hline Clinical stage & & & & & $0.028^{b}$ \\
\hline I - II & 18 & $7(38.9)$ & $11(61.1)$ & 7.96 & \\
\hline III - IV & 36 & $28(77.8)$ & $8(22.2)$ & & \\
\hline T classification & & & & & $0.882^{b}$ \\
\hline $\mathrm{T} 1-\mathrm{T} 2$ & 26 & $17(65.4)$ & $9(34.6)$ & 0.01 & \\
\hline $\mathrm{T} 3-\mathrm{T} 4$ & 28 & $18(64.3)$ & $10(35.6)$ & & \\
\hline $\begin{array}{l}\text { Lymphatic metas- } \\
\text { tasis }\end{array}$ & & & & & $0.001^{c}$ \\
\hline Yes & 27 & $25(92.6)$ & $2(7.4)$ & 18.27 & \\
\hline No & 27 & $10(37.0)$ & $17(63.0)$ & & \\
\hline
\end{tabular}

${ }^{a}$ Data are presented as No.(\%).

b Without significant differences.

c With significant differences. 


\subsection{TIMP-2 Expression}

Positive expression of TIMP-2 was associated with lymphatic metastasis, clinical staging and $\mathrm{T}$ classification. The positive rate of TIMP-2 expression in patients with lymphatic metastasis was significantly lower than those without it $\left(\chi^{2}=26.74, P=0.002\right)$, and the rate significantly reduced with increasing clinical stages and T classification $(\mathrm{P}<0.05)$. (Table 3, Figure 3).
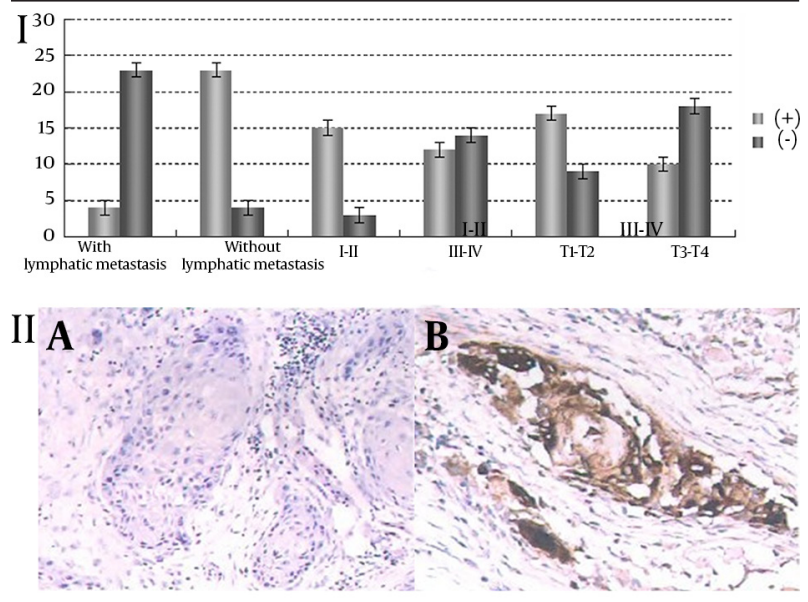

Figure 3. I: Association between TIMP-2 expression and lymphatic metastasis, clinical stages and T classification; II: Expression of TIMP-2 in lymph nodes. A: With lymphatic metastasis, negative expression (-); B: Without lymphatic metastasis, positive expression $(+)$.

\section{Discussion}

We found that VEGF, MMP-9 and TIMP-2 expression was associated with the clinical diagnosis, treatment and prevention of maxillofacial SCC. This study comprehensively revealed the effects of age, gender, lymphatic metastasis, clinical staging and T classification on the expression of VEGF, MMP-9 and TIMP-2. Meanwhile, as a retrospective study, the sample size was not sufficiently large, but the data was collected from more than three medical institutions. Therefore, we believe that the results are representative. Moreover, only the cases with primary foci and without metastases to other organs were included in this retrospective clinical study to assure its accuracy. However, a complicated staining method was used, which may involve operational errors because mucosae were sampled only once and did not allow a second staining. Regardless, the results herein are eligible and valid because the operational errors ranged between $(\mathrm{x} \pm 1)$.

VEGF, highly expressed in cancer cells, particularly in liver ones, essentially regulates the development of blood vessels, while promoting the migration of mononuclear macrophages $(11,12)$. Therefore, VEGF has relatively high positive expression in cancer cells with lymphatic metastasis.

MMP-9 is capable of regulating the release of VEGF (13), so VEGF is also highly expressed in cancer cells with high

\begin{tabular}{|c|c|c|c|c|c|}
\hline Related factor & $\mathbf{n}$ & Positive (+) & Negative (-) & $\chi^{2}$ & PValue \\
\hline Gender & & & & & $>0.999^{a}$ \\
\hline Male & 40 & $20(50.0)$ & $20(50.0)$ & 0.00 & \\
\hline Female & 14 & $7(50.0)$ & $7(50.0)$ & & \\
\hline Age & & & & & $0.902^{a}$ \\
\hline $26-50$ & 25 & $12(48.0)$ & $13(52.0)$ & 0.07 & \\
\hline $50-65$ & 29 & $15(51.7)$ & $14(48.3)$ & & \\
\hline Cancerous part & & & & & $0.935^{a}$ \\
\hline Tongue cancer & 6 & $5(83.3)$ & $1(16.7)$ & 0.23 & \\
\hline Floor-of-mouth cancer & 6 & $2(33.3)$ & $4(66.7)$ & & \\
\hline Cheek cancer & 33 & $24(72.7)$ & $9(27.3)$ & & \\
\hline Gum cancer & 10 & $7(70.0)$ & $3(30.0)$ & & \\
\hline Clinical stage & & & & & $0.028^{a}$ \\
\hline I - II & 18 & $15(83.3)$ & $3(16.7)$ & 12.00 & \\
\hline III - IV & 36 & $12(33.3)$ & $14(66.7)$ & & \\
\hline T classification & & & & & $0.048^{a}$ \\
\hline $\mathrm{T} 1-\mathrm{T} 2$ & 26 & $17(65.4)$ & $9(34.6)$ & 4.75 & \\
\hline $\mathrm{T} 3-\mathrm{T} 4$ & 28 & $10(35.7)$ & $18(64.3)$ & & \\
\hline Lymphatic metastasis & & & & & $0.002^{b}$ \\
\hline Yes & 27 & $4(14.8)$ & $23(85.2)$ & 26.74 & \\
\hline No & 27 & $23(85.2)$ & $4(14.8)$ & & \\
\hline
\end{tabular}

\footnotetext{
${ }^{\mathrm{a}}$ Without significant differences.

b with significant differences.
} 
expression of MMP-9, both of which are related with lymphatic metastasis. Its functions mainly depend on the effects of VEGF on vascular and lymphatic development and its involvement in phagocyte movement.

However, TIMP-2 can inhibit the high expression of MMPs (14), and render the latter unable to regulate the release of VEGF (15), thereby reducing the odds of lymphatic metastasis. In general, VEGF can directly regulate the development of blood and lymph vessels as well as phagocytes to induce lymphatic metastasis. MMP-9 can indirectly regulate VEGF to further induce lymphatic metastasis, while TIMP-2 can inhibit the expressions of MMP9 and VEGF by inhibiting MMPs. Lymphatic metastasis may finally occur in case of disequilibrium between the three. Therefore, the hypothesis of "genetic synergy" has been widely accepted.

In this study, brownish-yellow staining was correlated with positive expression of VEGF, MMP-9 and TIMP-2. Positive expression of VEGF and MMP-9 was correlated with lymphatic metastasis, the rates of which were both significantly higher in patients with lymphatic metastasis than those without it. In the meantime, the positive expression rate of MMP-9 reduced at earlier clinical stages. Moreover, positive expression of TIMP-2 had correlation with lymphatic metastasis, clinical staging and T classification, the rate of which in patients with lymphatic metastasis was significantly lower than those without it, and significantly decreased with increasing clinical stage and T classification.

In summary, pathogenesis studies concerning oral and maxillofacial SCC on the genetic level focus on the expressions of relevant genes to discover the mechanism of lymphatic metastasis, i.e. it is closely related to the expression of VEGF, MMP-9 and TIMP-2. Meanwhile, MMP9 and TIMP-2 could also affect the progression of cancer. Similarly, patients can also be treated based on the study of gene expression being the cutting edge of medical field, which necessitates more investigations.

\section{Acknowledgements}

We thank Dr. Lili Wang and PLA General Hospital (Beijing, China) for their kindly assistance in data observation and collection.

\section{Authors' Contribution}

Study concept and design: Min Li, Zhiying Wang. Analysis and interpretation of data: Min Li, Yang Xing, Jin Yu, Luming Tian, Dianming Zhang, Zengxi Xin. Critical revision of the manuscript for important intellectual content: Min Li, Zhiying Wang. Statistical analysis: Min Li.

\section{Financial Disclosure}

All coauthors declared no financial disclosure.

\section{Funding/Support}

All coauthors declared no funding/support.

\section{References}

1. Shakib K, Schrattenholz A, Soskic V. Stem cells in head and neck squamous cell carcinoma. BrJ Oral Maxillofac Surg. 2011;49(7):503-6.

2. Sterz CM, Kulle C, Dakic B, Makarova G, Bottcher MC, Bette M, et al. A basal-cell-like compartment in head and neck squamous cell carcinomas represents the invasive front of the tumor and is expressing MMP-9. Oral Oncol. 2010;46(2):116-22.

3. Liu CJ, Chang KW, Lin SC, Cheng HW. Presurgical serum levels of matrix metalloproteinase-9 and vascular endothelial growth factor in oral squamous cell carcinoma. Oral Oncol. 2009;45(10):920-5.

4. Roderfeld M, Rath T, Lammert F, Dierkes C, Graf J, Roeb E. Innovative immunohistochemistry identifies MMP-9 expressing macrophages at the invasive front of murine HCC. World J Hepatol. 2010;2(5):175-9.

5. Patel V, Marsh CA, Dorsam RT, Mikelis CM, Masedunskas A, Amorn phimoltham $\mathrm{P}$, et al. Decreased lymphangiogenesis and lymph node metastasis by mTOR inhibition in head and neck cancer. Cancer Res. 2011;71(22):7103-12.

6. Elahi M, Rakhshan V, Ghasemian NT, Moshref M. Prognostic value of transforming growth factor beta 1[TGF-beta1] and matrix metalloproteinase 9 [MMP-9] in oral squamous cell carcinoma. Biomarkers. 2012;17(1):21-7.

7. Suboj P, Babykutty S, Valiyaparambil Gopi DR, Nair RS, Srinivas P, Gopala S. Aloe emodin inhibits colon cancer cell migration/angiogenesis by downregulating MMP-2/9, RhoB and VEGF via reduced DNA binding activity of NF-kappaB. Eur J Pharm Sci. 2012;45(5):58191.

8. Hirano C, Nagata M, Noman AA, Kitamura N, Ohnishi M, Ohyama $\mathrm{T}$, et al. Tetraspanin gene expression levels as potential biomarkers for malignancy of gingival squamous cell carcinoma. Int J Cancer. 2009;124(12):2911-6.

9. Joshua B, Kaplan MJ, Doweck I, Pai R, Weissman IL, Prince ME, et al Frequency of cells expressing CD44, a head and neck cancer stem cell marker: correlation with tumor aggressiveness. Head Neck. 2012;34(1):42-9.

10. Mohtasham N, Babakoohi S, Shiva A, Shadman A, Kamyab-Hesari K, Shakeri MT, et al. Immunohistochemical study of p53, Ki-67, MMP-2 and MMP-9 expression at invasive front of squamous cell and verrucous carcinoma in oral cavity. Pathol Res Pract. 2013;209(2):110-4.

11. Heo SH, Choi YJ, Ryoo HM, Cho JY. Expression profiling of ETS and MMP factors in VEGF-activated endothelial cells: role of MMP-10 in VEGF-induced angiogenesis. J Cell Physiol.2010;224(3):734-42.

12. Chetty C, Lakka SS, Bhoopathi P, Rao JS. MMP-2 alters VEGF expression via alphaVbeta3 integrin-mediated PI3K/AKT signaling in A549 lung cancer cells. Int J Cancer. 2010;127(5):1081-95.

13. Kapoor V, Zaharieva MM, Das SN, Berger MR. Erufosine simultaneously induces apoptosis and autophagy by modulating the AktmTOR signaling pathway in oral squamous cell carcinoma. Cancer Lett. 2012;319(1):39-48.

14. Li M, Wu S, Liu Z, Zhang W, Xu J, Wang Y, et al. Arenobufagin, a bufadienolide compound from toad venom, inhibits VEGF-mediated angiogenesis through suppression of VEGFR-2 signaling pathway. Biochem Pharmacol.2012;83(9):1251-60.

15. Gao Q, Tong W, Luria JS, Wang Z, Nussenbaum B, Krebsbach PH Effects of bone morphogenetic protein-2 on proliferation and angiogenesis in oral squamous cell carcinoma. Int J Oral Maxillofac Surg. 2010;39(3):266-71 